\title{
The variation in transparency of amniotic membrane used in ocular surface regeneration
}

Article

Accepted Version

proof document ahead of publication

Connon, C. J., Doutch, J., Chen, B., Hopkinson, A., Mehta, J., Nakamura, T., Kinoshita, S. and Meek, K. (2010) The variation in transparency of amniotic membrane used in ocular surface regeneration. British Journal of Ophthalmology, 94 (8). pp. 1057-1061. ISSN 0007-1161 doi:

https://doi.org/10.1136/bjo.2008.153064 Available at https://centaur.reading.ac.uk/1680/

It is advisable to refer to the publisher's version if you intend to cite from the work. See Guidance on citing.

To link to this article DOI: http://dx.doi.org/10.1136/bjo.2008.153064

Publisher: BMJ Publishing Group

All outputs in CentAUR are protected by Intellectual Property Rights law, including copyright law. Copyright and IPR is retained by the creators or other copyright holders. Terms and conditions for use of this material are defined in the End User Agreement. 


\section{CentAUR}

Central Archive at the University of Reading

Reading's research outputs online 


\section{Queries for Author}

Journal: British Journal of Ophthalmology

Paper: bj153064

Title: The variation in transparency of amniotic membrane used in ocular surface regeneration

The proof of your manuscript appears on the following page(s).

Please note that this is a galley proof and the layout of the article may change before publication. Please read the manuscript carefully, checking for accuracy, verifying the reference order and double-checking figures and tables. When reviewing your page proof please keep in mind that a professional copyeditor edited your manuscript to comply with the style requirements of the journal. This is not an opportunity to alter, amend or revise your paper; it is intended to be for correction purposes only.

During the preparation of your manuscript for publication, the questions listed below have arisen (the query number can also be found in the gutter close to the text it refers to). Please attend to these matters and return the answers to these questions when you return your corrections.

Please note, we will not be able to proceed with your article and publish it in print if these queries have not been addressed.

\begin{tabular}{|l|l|}
\hline $\begin{array}{l}\text { Query } \\
\text { Reference }\end{array}$ & Query \\
\hline 1 & $\begin{array}{l}\text { Please check that the full details provided in the correspondence address are } \\
\text { correct }\end{array}$ \\
\hline 2 & I have updated reference 6. Please check. \\
\hline
\end{tabular}

If you are happy with the proof as it stands, please email to confirm this. Changes that do not require a copy of the proof can be sent by email (please be as specific as possible).

Email: paul@paulsen.demon.co.uk

If you have any changes that cannot be described easily in an email, please mark them clearly on the proof and email a scan of the changes by replying to the eProof email or by fax: $+44(0) 8700515632$. 


\title{
The variation in transparency of amniotic membrane used in ocular surface regeneration
}

\author{
C J Connon, ${ }^{1}$ J Doutch, ${ }^{2}$ B Chen, ${ }^{1}$ A Hopkinson, ${ }^{3}$ J S Mehta, ${ }^{4}$ T Nakamura, ${ }^{5}$ \\ S Kinoshita, ${ }^{5}$ K M Meek ${ }^{2}$
}

${ }^{1}$ School of Pharmacy, University of Reading, Reading, UK; ${ }^{2}$ School of Optometry \& Vision Sciences, Cardiff University, Cardiff, UK; ${ }^{3}$ Division of Ophthalmology \& Visual Sciences, Queen's Medical Centre, Nottingham, UK;

${ }^{4}$ Singapore National Eye Centre, Singapore; ${ }^{5}$ Department of Ophthalmology, Kyoto Prefectural University of Medicine, Kyoto, Japan

Correspondence to: Dr C J Connon, School of Pharmacy, University of Reading. $\mathrm{Rm}$ 108, Hopkins Building Whiteknights, PO Box 228,

Reading RG6 6UB, UK

c.j.connon@reading.ac.uk

Accepted 9 February 2009 Published Online First 19 March 2009

\section{ABSTRACT}

Background/aims: Scant consideration has been given to the variation in structure of the human amniotic membrane (AM) at source or to the significance such differences might have on its clinical transparency. Therefore, we applied our experience of quantifying corneal transparency to AM.

Methods: Following elective caesarean, AM from areas of the fetal sac distal and proximal (ie, adjacent) to the placenta was compared with freeze-dried AM. The transmission of light through the AM samples was quantified spectrophotometrically; also, tissue thickness was measured by light microscopy and refractive index by refractometry.

Results: Freeze-dried and freeze-thawed AM samples distal and proximal to the placenta differed significantly in thickness, percentage transmission of visible light and refractive index. The thinnest tissue (freeze-dried AM) had the highest transmission spectra. The thickest tissue (freeze-thawed AM proximal to the placenta) had the highest refractive index. Using the direct summation of fields method to predict transparency from an equivalent thickness of corneal tissue, AM was found to be up to $85 \%$ as transparent as human cornea.

Conclusion: When preparing AM for ocular surface reconstruction within the visual field, consideration should be given to its original location from within the fetal sac and its method of preservation, as either can influence corneal transparency.

For several years in vivo and in vitro-based evidence has demonstrated the ability of amniotic membrane to provide a natural substrate upon which cells can grow. ${ }^{1-3}$ Subsequently, the human amniotic membrane (AM) is now firmly established as an important adjunct for ocular surface reconstruction across a broad spectrum of conditions ${ }^{4}$ where it is often directly applied as a patch or a graft. For more serious conditions, such as limbal stem cell deficiency, AM taken from the fetal sac has also been employed as a substrate on to which donor corneal epithelial progenitor (limbal) cells are expanded, forming tissue-engineered constructs suitable for surgical application. ${ }^{5}$ However, such therapeutic applications of AM often result in its postoperative positioning within the visual field and sometimes, as in limbal stem cell transplantation, for prolonged or indefinite periods. In such cases, the question of AM's transparency becomes crucial.

AM is the most structurally robust of the fetal membranes ${ }^{6}$ consisting of a single layer of epithelial cells on a thick basement membrane which in turn lies upon layers of collagenous tissue interspersed with mesenchymal cells maintaining the mechanical integrity of the tissue. ${ }^{7}$ Interstitial collagens (types I and III) predominate and form parallel bundles of collagen fibrils that produce a scaffold similar in ultrastructual organisation to that seen within the stroma of the cornea. ${ }^{8}$ The AM stroma however is considerably thinner than that of the human cornea, and when used therapeutically, the amniotic epithelia are lost and replaced by native corneal epithelia. ${ }^{9}$ However, AM stroma can persist in its native form for many months following transplantation under specific conditions.

Recently we have shown that there exists a significant variation in structure between different areas of the amniotic sac, ${ }^{10}{ }^{11}$ but despite the increasing use of AM in ophthalmic therapeutic applications there is presently little consideration given to the importance such variation in membrane structure may have on subsequent therapeutic effect, especially clinical transparency. Previously, we have investigated the fine structural organisation of collagen fibrils within wounded and normal corneas and successfully related changes in fibril organisation to corneal transparency. ${ }^{12-19}$ Thus, considering the previously observed similarities in structure between AM stroma and corneal stroma, we have now applied our expertise in understanding corneal transparency to the transparency of clinically relevant AM.

\section{MATERIALS AND METHODS}

\section{Collection and storage of human amniotic} membrane

Following elective Caesarean section at term, unlinked anonymised samples of amniotic membrane were taken from fetal sac membranes adjoining, but not overlaying, the placenta. Fetal membranes overlaying the placenta were not included, as they are not commonly used in stem cell transplantation, the technique most strongly associated with the positioning of AM within the visual field for prolonged or indefinite periods. Fetal sac membranes from six patients were collected from the Department of Obstetrics and Gynaecology, Queen's Medical Centre, Nottingham, UK, after full local ethics committee approval and in compliance with the Declaration of Helsinki. The fetal membranes were prepared in accordance with a previously published procedure. ${ }^{11}$ First, the chorion was separated manually from the amnion and discarded; the remaining AM was washed with phosphate-buffered saline (PBS) containing antibiotics (5 $\mathrm{ml}$ of $0.5 \%$ levofloxacin) to remove blood. Persistent blood-stained AM edges were dissected away and not used. Then, 
under sterile conditions, samples $(4 \mathrm{~cm} \times 4 \mathrm{~cm})$ of $\mathrm{AM}$ were taken from areas adjacent to the placental disc (proximal amnion) and approximately $10 \mathrm{~cm}$ from the placental disc (distal amnion). These samples were chosen from areas of the AM which were coherent and translucent. The dissected AM samples were stored at $-80^{\circ} \mathrm{C}$ in $\mathrm{PBS}$. These samples were thawed before further examination and subsequently termed freeze-thawed AM. It has previously been confirmed that there is no difference between fresh and frozen AM in terms of clinical efficacy. ${ }^{20}$

Four further placentas, providing samples for freeze-dried AM, were received either as a gift (Dr T Nakamura, Kyoto Prefectural University of Medicine, Japan having been prepared according to their published method ${ }^{21}$ ) or supplied commercially (Acelagraft, Celgene Cellular Therapeutics, New Jersey).

\section{Transmission measurements}

The freeze-thawed amniotic samples were incubated in Dispase (Gibco, Grand Island, New York) at $37^{\circ} \mathrm{C}$ for $2 \mathrm{~h}$ and the epithelium removed by scraping. Both the freeze-thawed and freeze-dried samples were incubated in PBS at room temperature for $2 \mathrm{~h}$ before the transmission of light through each sample was measured. Each sample of AM in PBS was flattened and held securely between the two glass plates of a $35 \mathrm{~mm}$ slide mount. The glass slide mount maintained the AM in a hydrated state with no wrinkles or air bubbles. Each mounted sample was then placed, in turn, within a spectrophotometer (PYE Unicom, SP8-100), and percentage transmission was recorded through the visible spectrum $(400-700 \mathrm{~nm})$. The process was repeated three times for each sample, each time exposing a different area of tissue to the incident light beam. The transmission values were zeroed by subtracting the glass slide mount with PBS alone.

\section{Refractive index measurements}

Following transmission measurements, the refractive index of each AM sample was quantified using a bench-top Abbe 60 Series Refractometer (Bellingham and Stanley, Tunbridge Wells, UK). The refractive index was measured from three different areas of each sample independently by two observers, and the average value calculated.

\section{Thickness measurements}

Immediately following transmission measurements a small area $\left(10 \mathrm{~mm}^{2}\right)$ from each sample was embedded in Tissue Tek (Fisher Scientific, Loughborough, UK), snap-frozen in liquid nitrogen and stored at $-80^{\circ} \mathrm{C}$. Cryo-sections $(7 \mu \mathrm{m})$ were then stained with haematoxylin and eosin, and thickness measurements across the AM recorded using a calibrated microscope with digital camera (Zeiss, Jena, Germany; Axioskop 2). To compensate for the natural heterogeneity of AM structure three serial sections were taken from three regions of increasing depth through each embedded sample, 10 measurements were taken from each section, and the results were averaged.

\section{Predicted transparency calculations}

Transparency through corneal stroma can be predicted using an established model, the direct summation of fields for light scattering by fibrils. ${ }^{12}{ }^{22}{ }^{23}$ In this study we applied the same model to predict transparency through corneal stroma with a thickness artificially reduced to that of AM. This facilitated a direct comparison in transmission spectra between the cornea (predicted) and AM (actual) by normalising for tissue thickness.
A
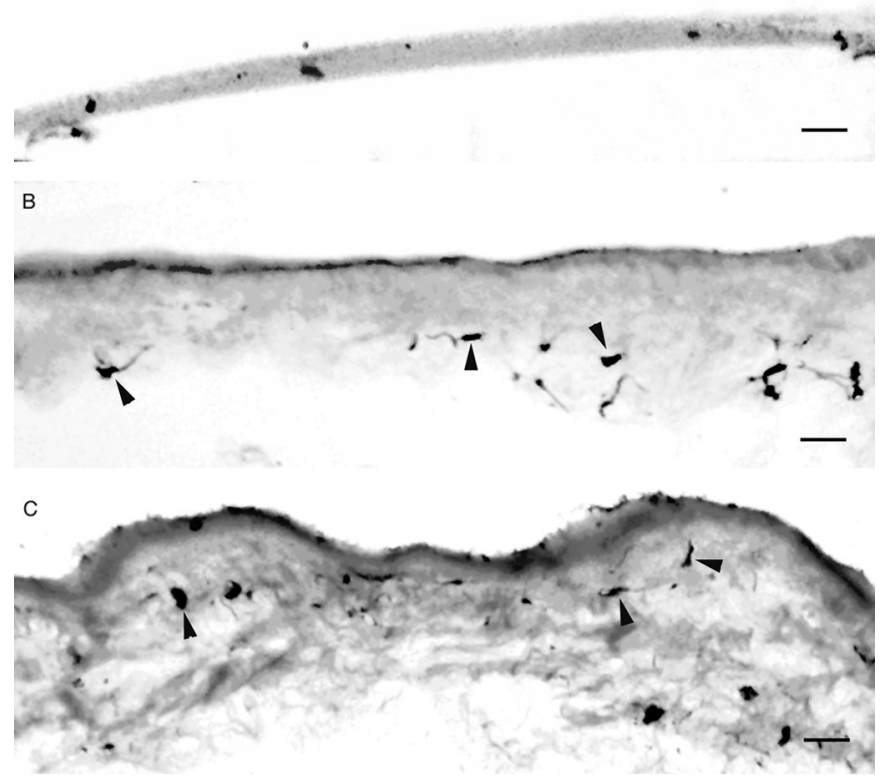

Figure 1 Freeze-dried amniotic membrane (AM). This lacks the presence of epithelial or stromal cells and is of uniform thickness (A). Freeze-thawed AM is uneven in its thickness, and AM collected distal to the placental $(B)$ is thinner than $A M$ collected proximal/adjacent to the placenta (C). While epithelial cells have been successfully removed by enzyme treatment followed by scraping, stromal cells persist (arrows). However, these cells are likely to be devitalised following the freezethaw process. Scale bars $=20 \mu \mathrm{m}$.

Briefly, assuming there is no absorption, the fraction of light transmitted undeviated through the cornea is related to the total scattering cross-section per fibril per unit length, $\sigma$, by the equation $F(\lambda)=e^{-\rho \sigma t}$, where $t$ is the thickness of the stroma, $\rho$ is the bulk number density of fibrils in the stroma, and $\sigma$ is the scattering cross-section, a function of the following, (1) the size of the fibrils, (2) the packing of the fibrils, (3) the refractive indices of the hydrated fibrils and hydrated interfibrillar matrix and (4) wavelength $(\lambda)$. $t$ was calculated from our light

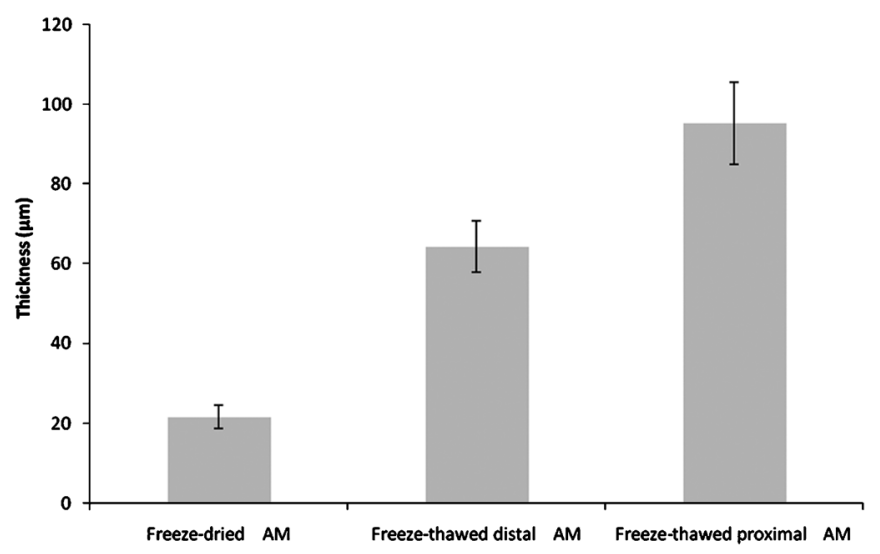

Figure 2 Thickness measurements of freeze-dried, freeze-thawed distal and freeze-thawed proximal amniotic membrane (AM) taken from the light micrographs. Proximal AM was significantly found to be the thickest, while freeze-dried AM was found to be significantly the thinnest. Error bars correspond to the standard error. 
Figure 3 Percentage transmission of visible light compared through freezedried, freeze-thawed distal and freezethawed proximal amniotic membrane (AM). The freeze-thawed proximal AM had the lowest transmission spectra, whereas the freeze-dried had the highest. Error bars correspond to the standard error.

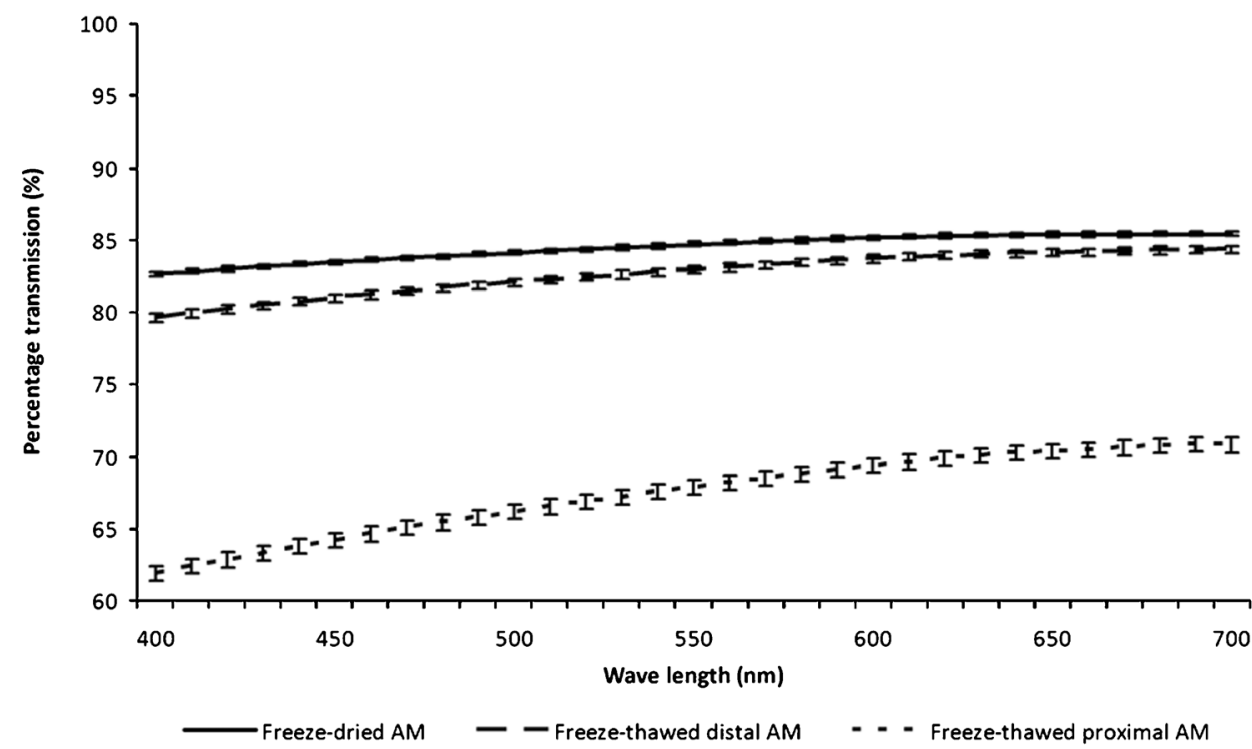

microscopy measurements of AM, the size and packing of corneal collagen fibrils were taken from representative published electron micrographs of human corneal stroma, ${ }^{24}$ and the refractive index of the fibrils and interfibrillar matrix was taken from previously published data. ${ }^{25}$

\section{RESULTS}

Light microscopy confirmed that the epithelial cells had been successfully removed from the freeze-thawed AM and that none were present on the surface of freeze-dried AM prior to transmission measurements (fig 1). The freeze-dried, freezethawed distal and freeze-thawed proximal AM had a mean thickness of 21.6 (SD 5.6) $\mu \mathrm{m}, 64.3$ (20.9) and $95.3 \mu \mathrm{m}$ (27.9) respectively. The freeze-dried AM was significantly thinner than the freeze-thawed AM $(p<0.01$, Student $t$ test). Within the freeze-thawed AM samples, thickness was significantly greater in areas proximal to the placenta when compared with AM collected from areas distal to the placenta $(\mathrm{p}<0.01$, Student t test) (fig 2).

Transparency (percentage transmission of visible light) differed significantly between the three types of AM investigated (freeze-dried, freeze-thawed proximal and freeze-thawed distal). Transparency increased in line with tissue thickness, the freeze-dried AM (thinnest) having the highest transmission spectra and the freeze-thawed proximal AM (thickest) having the lowest transmission spectra (fig 3).

The freeze-dried, freeze-thawed distal and freeze-thawed proximal AM had a mean refractive index of 1.335 (0.001), $1.334(0.002)$ and $1.357(0.002)$ respectively. There was no significant difference between the refractive index of freezedried and freeze-thawed distal AM both having a similar refractive index to water (1.333). The refractive index of freeze-thawed proximal AM was significantly higher than the freeze-thawed distal AM ( $p<0.001$, Student t test) and much nearer to the refractive index of the corneal stroma $(1.375)^{25}$ (fig 4).

The direct summation of fields method facilitated a comparison in transparency between corneal tissue of different thicknesses (fig 5). The values of the thicknesses used were taken from the AM thickness measurements by light microscopy (fig 2). By comparing the predicted levels of transparency with the actual AM transmission spectra, shown in fig 3, freezedried, freeze-thawed distal and freeze-thawed proximal AM were calculated to be $85 \%, 83 \%$ and $68 \%$ as transparent as the
Figure 4 Refractive index compared between freeze-dried, freeze-thawed distal and freeze-thawed proximal amniotic membrane (AM). No significant difference was observed between freezedried and freeze-thawed distal AM, both similar to water. Freeze-thawed proximal AM had a significantly high refractive index than the other types of AM. *Refractive index values of human cornea and water were taken from published data. ${ }^{25}$ Error bars correspond to the standard error.

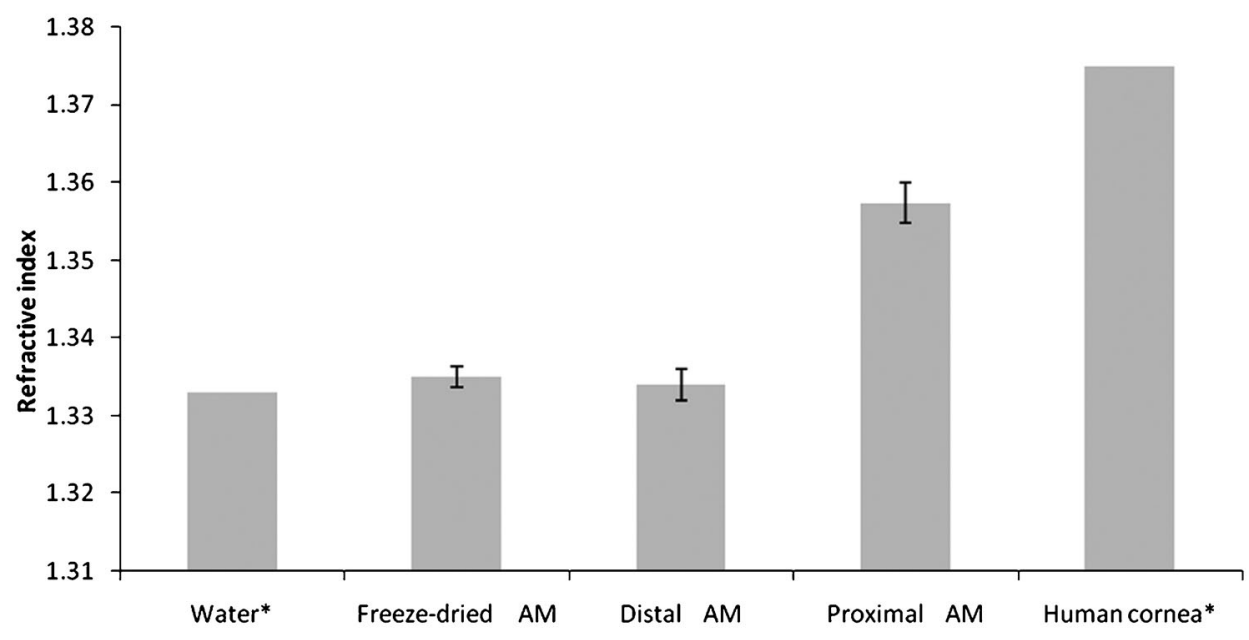


Figure 5 Predicted transmission of visible light through cornea at various thicknesses using the direct summation of fields method. The graph clearly shows a decrease in transparency with increasing tissue thickness. Thickness values correspond to the measured thickness of freeze-dried, freeze-thawed distal and freeze-thawed proximal AM. A comparison between these spectra and measured spectra through amniotic membrane (AM) (fig 3) facilitates a direct evaluation of transparency between cornea and AM by normalising for tissue thickness.

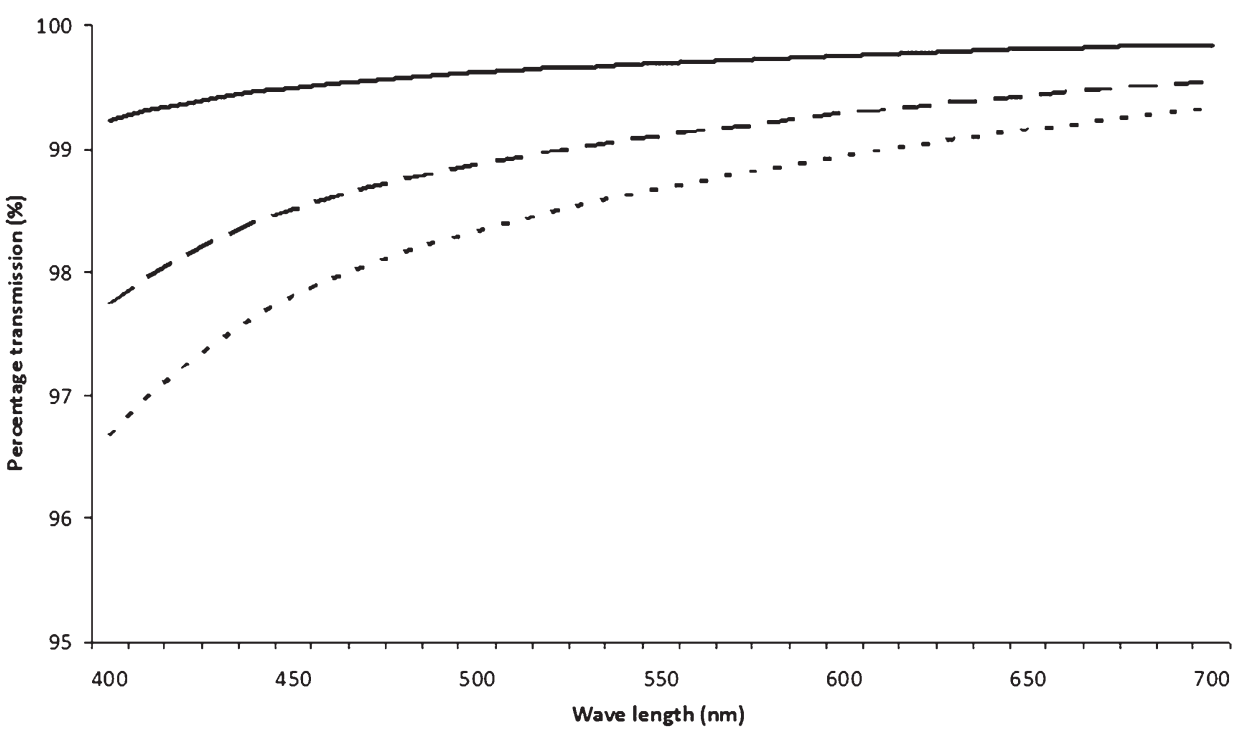

- Freeze-dried AM - Cornea of equivalent thickness to: - Freeze-thawed distal AM Freeze-thawed proximal AM human cornea respectively once normalised for stromal thickness.

\section{DISCUSSION}

The results suggest that significant variations in the optical properties of AM exist. We have shown that preservation and sampling protocol can influence both the transmission of visible light and refractive index of AM used for ocular surface regeneration. The divergence in measured transparency between freeze-dried AM and freeze-thawed AM, despite having a similar refractive index, is most likely explained by differences in tissue thickness, since, when normalised for thickness, there was very little difference between the subsequent predicted corneal transparencies. However, the relative smoothness of the freeze-dried AM surface and complete absence of epithelial cells would have also reduced the scatter of incident light increasing its transparency.

Interestingly, the smallest relative difference in refractive index between human cornea and AM was shown by the freezethawed proximal sample. This may have some clinical relevance, as the larger the difference in refractive index between cornea and transplanted AM, the greater the chance of scatter at the interface between the two tissues would be. If this were the case and despite its lower transparency, freezethawed proximal AM may be more suitable for the packing of deep corneal wounds, such as ulcers, especially if we consider that AM can persist unaltered within the corneal stroma for 12 months. $^{8}$

Using AM for ocular surface reconstruction within the visual field, tissue taken from an area of the amniotic sac distal to the placenta offers the greatest transparency. However, freeze-dried AM preservation provides an increased level of transparency over a freeze-thaw method of preservation.

Funding: We would like to acknowledge funding support from the NC3R, Diawa Anglo-Japanese Foundation and JSPS Furusato Award (CJC). KMM is a Royal Society/ Wolfson Foundation Merit Award holder.

Competing interests: None.

Ethics approval: Ethics approval was provided by Queen's Medical Centre, Nottingham.

\section{REFERENCES}

1. Meller D, Pires RT, Tseng SC. Ex vivo preservation and expansion of human limbal epithelial stem cells on amniotic membrane cultures. Br J Ophthalmol 2002;86:46371.

2. Schwab IR. Cultured corneal epithelia for ocular surface disease. Trans Am Ophthalmol Soc 1999;97:891-986.

3. Koizumi N, Inatomi T, Quantock AJ, et al. Amniotic membrane as a substrate for cultivating limbal corneal epithelial cells for autologous transplantation in rabbits. Cornea 2000;19:65-71.

4. Gomes JA, Romano A, Santos MS, et al. Amniotic membrane use in ophthalmology. Curr Opin Ophthalmol 2005;16:233-40.

5. Tsai RJ, Li LM, Chen JK. Reconstruction of damaged corneas by transplantation of autologous limbal epithelial cells. N Engl J Med 2000;343:86-93.

6. Moore RM, Mansour JM, Redline RW, et al. The physiology of fetal membrane rupture: insight gained from the determination of physical properties. Placenta 2006;27:1037-51.

7. Malak TM, Ockleford CD, Bell SC, et al. Confocal immunofluorescence localization of collagen types I, III, IV, V and VI and their ultrastructural organization in term human fetal membranes. Placenta 1993;14:385-406.

8. Connon CJ, Nakamura T, Quantock AJ, et al. The persistence of transplanted amniotic membrane in corneal stroma. Am J Ophthalmol 2006;141:190-2

9. Nubile M, Dua HS, Lanzini TE-M, et al. Amniotic membrane transplantation for the management of corneal epithelial defects: an in vivo confocal microscopic study. $\mathrm{Br} J$ Ophthalmol 2008:92:54-60.

10. Connon CJ, Nakamura T, Hopkinson A, et al. The biomechanics of amnion rupture an x-ray diffraction study. PLOS ONE 2007;2:e1147.

11. Hopkinson A, Mclntosh RS, Tighe PJ, et al. Amniotic membrane for ocular surface reconstruction: donor variations and the effect of handling on TGF-\{beta $\}$ content. Invest Ophthalmol Vis Sci 2006:47:4316-22.

12. Connon CJ, Marshall J, Patmore AL, et al. Persistent haze and disorganization of anterior stromal collagen appear unrelated following phototherapeutic keratectomy. J Refract Surg 2003;19:323-32.

13. Connon CJ, Meek KM. Organization of corneal collagen fibrils during the healing of trephined wounds in rabbits. Wound Repair Regen 2003:11:71-8.

14. Meek K, Leonard D, Connon C, et al. Transparency, swelling and scarring in the corneal stroma. Eye 2003;17:927-36.

15. Connon CJ, Meek KM. The structure and swelling of corneal scar tissue in penetrating full-thickness wounds. Cornea 2004;23:165-71.

16. Connon CJ, Meek KM, Kinoshita S, et al. Spatial and temporal alterations in the collagen fibrillar array during the onset of transparency in the avian cornea. Exp Eye Res 2004;78:909-15.

17. Meek KM, Elliott GF, Sayers Z, et al. Interpretation of the meridional x-ray diffraction pattern from collagen fibrils in corneal stroma. J Mol Biol 1981;149 477-88.

18. Meek KM, Leonard DW. Ultrastructure of the corneal stroma: a comparative study. Biophys J 1993;64:273-80.

19. Meek KM, Ouantock AJ. The use of $X$-ray scattering techniques to determine corneal ultrastructure. Prog Retin Eye Res 2001;20:95-137.

20. Adds PJ, Hunt CJ, Dart JKG. Amniotic membrane grafts, "fresh" or frozen? A clinical and in vitro comparison. $\mathrm{Br} \mathrm{J}$ Ophthalmol 2001:85:905-7. 
21. Nakamura T, Yoshitani M, Rigby $\mathrm{H}$, et al. Sterilized, freeze-dried amniotic membrane: a useful substrate for ocular surface reconstruction. Invest Ophthalmol Vis Sci 2004;45:93-9.

22. Freund DE, McCally RL, Farrell RA. Direct summation of fields for light scattering by fibrils with applications to normal corneas. Appl Opt 1986;25:2739
23. McCally RL, Freund DE, Zorn A, et al. Light-scattering and ultrastructure of healed penetrating corneal wounds. Invest Ophthalmol Vis Sci 2007;48:157-65.

24. Komai Y, Ushiki T. The three-dimensional organization of collagen fibrils in the human cornea and sclera. Invest Ophthalmol Vis Sci 1991:32:2244-58.

25. Leonard DW, Meek KM. Refractive indices of the collagen fibrils and extrafibrillar material of the corneal stroma. Biophys J 1997;72:1382-7. 